\title{
Restoring betatron phase coherence in a beam-loaded laser-wakefield accelerator
}

\author{
A. Koehler®, ${ }^{1, *}$ R. Pausch $\odot,{ }^{1}$ M. Bussmann $\odot,{ }^{2,1}$ J. P. Couperus Cabadağ $\odot,{ }^{1}$ A. Debus $\odot,{ }^{1}$ \\ J. M. Krämer®, ${ }^{1}$ S. Schöbel $\odot,{ }^{1,3}$ O. Zarini, ${ }^{1}$ U. Schramm $\odot,{ }^{1,3}$ and A. Irman ${ }^{1}$ \\ ${ }^{1}$ Helmholtz-Zentrum Dresden-Rossendorf, Bautzner Landstrasse 400, 01328 Dresden, Germany \\ ${ }^{2}$ Center for Advanced Systems Understanding, 02826 Görlitz, Germany \\ ${ }^{3}$ Technische Universität Dresden, 01062 Dresden, Germany
}

(Received 9 July 2021; accepted 16 August 2021; published 20 September 2021)

\begin{abstract}
Matched beam loading in laser wakefield acceleration, characterizing the state of flattening the accelerating electric field along the bunch, leads to the minimization of energy spread at high-bunch charges. Here, we experimentally demonstrate by independently controlling injected charge and accelerating gradients, using the self-truncated ionization injection scheme, that minimal energy spread coincides with a reduction of the normalized beam divergence. With the simultaneous confirmation of the micrometer-small beam radius at the plasma exit, deduced from betatron radiation spectroscopy, we attribute this effect to the minimization of chromatic betatron decoherence. These findings are supported by rigorous three-dimensional particle-in-cell simulations tracking self-consistently particle trajectories from injection, acceleration until beam extraction to vacuum. We conclude that beam-loaded laser wakefield acceleration enables highest longitudinal and transverse phase space densities.
\end{abstract}

DOI: 10.1103/PhysRevAccelBeams.24.091302

\section{INTRODUCTION}

The concept of laser wakefield acceleration (LWFA) exploits ultrahigh accelerating field gradients of up to a few hundred Gigavolt-per-meter generated in the wake of a high-intensity laser pulse as it propagates through an optically transparent plasma [1,2]. Electron bunches can thus be accelerated to $\mathrm{GeV}$ energies within centimeters [3]. Beam quality with respect to bunch charge, energy bandwidth, emittance, and pulse-to-pulse stability has improved substantially during the last decade and is closely linked to a variety of controlled electron injection techniques [4-9]. Only recently it was demonstrated that laser-plasma accelerators can be tailored for minimum energy spread at highbunch charges by reshaping the local accelerating field via matched beam loading [9-12]. This combination of high charge, essential for the beam loading regime, and the short bunch duration in the range of $10 \mathrm{fs}$ [13-17] results in high peak-current beams exceeding $10 \mathrm{kA}$. Future applications, such as high-field $\mathrm{THz}$ sources [18], laboratory-size beam-driven plasma accelerators [19-21] and compact

\footnotetext{
*a.koehler@hzdr.de

Published by the American Physical Society under the terms of the Creative Commons Attribution 4.0 International license. Further distribution of this work must maintain attribution to the author(s) and the published article's title, journal citation, and DOI.
}

free-electron lasers [22-26], will benefit from such compact and further improved high-brightness electron sources.

For realizing such high-quality beams, not only beam energy spread but also transverse emittance, dominated by beam divergence $[27,28]$, has to be minimized. In transverse phase space $\left(x, p_{x}\right)$, a polychromatic bunch can be divided into slices representing different energy classes, schematically illustrated in Fig. 1. The wakefield provides a strong linear focusing force [29], such that off axis electrons perform transverse (betatron) oscillations around the beam axis and emit radiation while being accelerated [2,30-33]. Due to the coupling of longitudinal acceleration and linear focusing, the relativistic mass increase of the electrons results in energy-dependent angular frequencies, amplitudes, and phases. Hence, the beam undergoes significant envelope oscillations [Fig. 1(c)] [34,35]. As a consequence, the betatron oscillations can eventually lose phase coherence for a polychromatic beam. For energychirped bunches typical in LWFA, a phase difference $\Delta \phi \geq \pi$ between the highest and the lowest energy slice leads to full decoherence and maximizes the occupied phasespace area, i.e., in beam divergence and size. Only for $\Delta \phi<\pi$, the phase space is not filled entirely and thus the beam can be coupled out either at minimum beam size or divergence. Simulation studies suggest that a minimum phase difference is only achievable for a very short injection duration that results in both a small initial phase spread and a similar accelerating field thus leading to a small final energy spread $[34,35]$. This, however, has the disadvantage of only providing small charges of a few picocoulomb. 


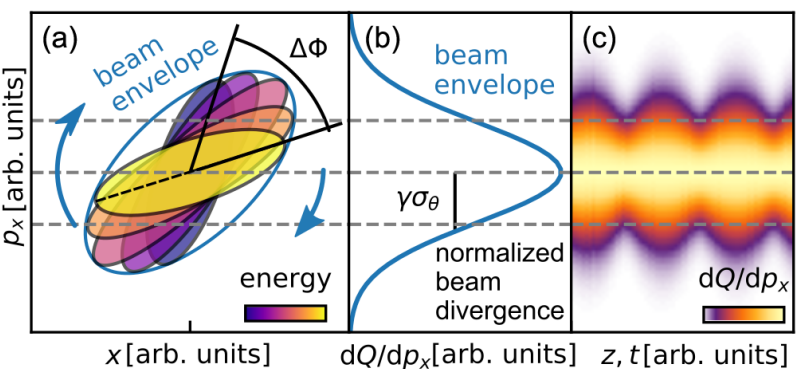

FIG. 1. Beam decoherence: (a) illustrates the transverse phase space of a bunch with finite energy spread consisting of many slices of different electron energy which rotate in phase space with the energy-dependent betatron frequency directed by the focusing force. The beam envelope encircles the sum of all slices, (b) shows the projection $\mathrm{d} Q / \mathrm{d} p_{x}$ of the beam on $p_{x}$, which is typically recorded in experiments and used for determining the normalized beam divergence $\gamma \sigma_{\Theta}$, and (c) shows the timedependence that is caused by the phase-space rotation of the beam envelope. When the slices in (a) span over $\Delta \phi \geq \pi$, full decoherence is reached and the modulation in (c) vanishes. For $\Delta \phi<\pi$, the beam can be extracted at a phase with reduced momentum spread and small $\sigma_{\Theta}$.

Here, we report on experimental findings in the beam loading regime that show improved transverse beam quality by restoring beam coherence while providing a high charge. Despite having hundreds of picocoulomb charges, control on the beam coherence decreases beam divergence by about $20 \%$. At the matched beam loading condition, a balance between the bunch's self-fields and the accelerating field of the wakefield is reached, resulting in a constant longitudinal accelerating field along the bunch [36,37]. As a result, all electrons within the bunch experience the same accelerating field so that no energy bandwidth is added during the acceleration process. Completing the examination of $\left(x, p_{x}\right)$, betatron radiation spectroscopy confirms a beam radius of $<0.7 \mu \mathrm{m}$ at the plasma exit. The simultaneous measurements of the small beam size and the minimized divergence demonstrate an increased transverse beam quality and reduce emittance, facilitating beam transport and novel applications. Since beam loading can reduce the energy spread, the control of this spread provides a unique opportunity to study electron beam coherence. For further investigating the coherence-restoring process close to the experimental conditions, we performed systematic high resolution, self-consistent particle-in-cell (PIC) simulations that confirmed our findings. To completely study the coherence restoration, we examined electron ionization, trapping, and acceleration from low to high energies in detail. The effect of space charge during field-free propagation to the detector system is assessed by tracing particles with a particle tracker code. The combination of experimental and simulation data provides valuable insight into the evolution of the phase space and thus enhances the understanding of coherence and decoherence in high-charge LWFA.

\section{EXPERIMENTAL SETUP}

Experiments were performed with the Dresden laser acceleration source (DRACO) laser system [38] at the Helmholtz-Zentrum Dresden - Rossendorf (HZDR). Figure 2(a) shows a schematic of the setup reproducing conditions as in Ref. [10]. Laser pulses of 30 fs full-width at half-maximum (FWHM) duration with $2.5 \mathrm{~J}$ energy on target were focused with an off axis parabolic mirror $(f / 20)$ to a vacuum focal spot size of $20 \mu \mathrm{m}$ (FWHM) yielding a normalized laser intensity of $a_{0} \simeq 2.6$. The Strehl ratio of (a)

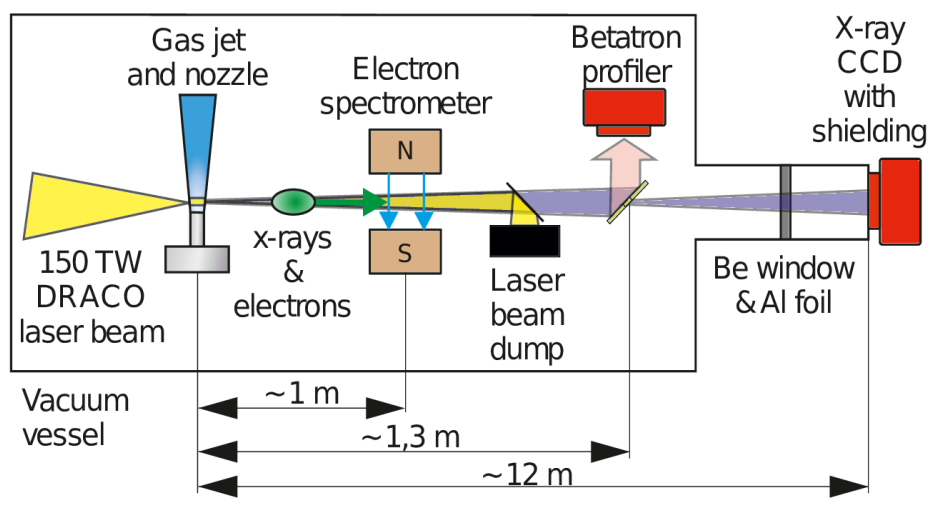

(b)

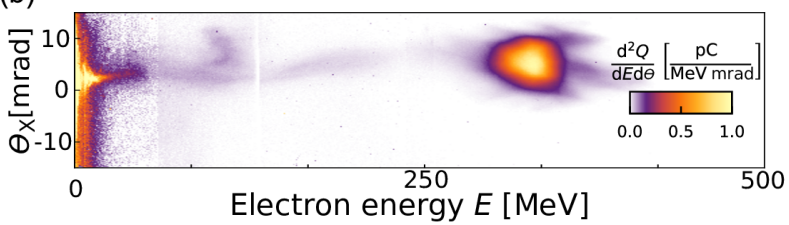

(c)

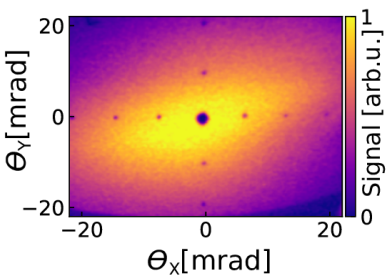

(d)

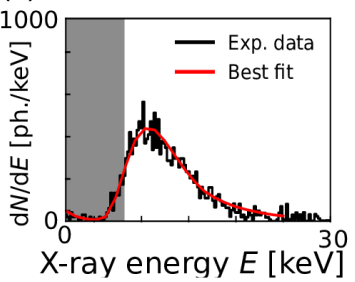

FIG. 2. The experimental setup is presented in (a) the laser is focused on a gas jet and drives a wakefield. Accelerated electrons are energy-analyzed in a magnet spectrometer, (b) illustrates a typical angle-resolved energy spectrum of electrons. An aluminum foil reflects residual laser light to a beam dump behind the spectrometer. As shown in (c), the betatron profiler intercepts the angular profile of the betatron radiation and on axis betatron radiation passes through a hole in the scintillator which is indicated by the black circular area in the center. Only the on axis part of the betatron spectra is detected by the x-ray camera, which is separated from the interaction chamber by a beryllium window. The reconstructed betatron spectrum is shown in (d). The gray area indicates photon energies that are not transmitted by the beam line. An aluminum filter foil attenuates the betatron flux. 
the wavefront-corrected and transversely symmetric beam was measured to be 0.9. The laser beam was focused $1.5 \mathrm{~mm}$ beyond the center of a $3 \mathrm{~mm}$ long de Laval gas nozzle. The nozzle was operated with $\mathrm{He}-\mathrm{N}_{2}$ gas mixtures containing $0.2 \%-1.5 \%$ of $\mathrm{N}_{2}$. The gas density profile at $1.5 \mathrm{~mm}$ above the nozzle exit, i.e., the laser beam axis, exhibits a $1.6 \mathrm{~mm}$ flat-top region [39]. Electrons were injected into the wakefield using self-truncated ionization injection $[10,40,41]$, which enabled stable and reproducible shots with high charges of up to $500 \mathrm{pC}$ (within FWHM). The contribution of other injection mechanisms to the charge was found to be negligible in the experiment [10]. In order to measure the energy distribution and divergence, a $40 \mathrm{~cm}$ long permanent magnet dipole, positioned downstream of the plasma accelerator, dispersed the accelerated electrons to a set of charge calibrated scintillator screens (Konica Minolta OG 400) $[38,42]$.

A back-illuminated, deep depletion x-ray CCD (Princeton Instruments Pixis-XO 400BR) with $1340 \times$ 400 pixel recorded betatron radiation emitted from the LWFA process. The camera was placed inside a dedicated radiation-shielded area $12 \mathrm{~m}$ downstream of the plasma target, covering a solid angle of $1.5 \times 0.8 \mathrm{mrad}$. A $76 \mu \mathrm{m}$ thick beryllium window sealed the charge coupled device (CCD) chip, allowing for cooling and background noise reduction. An aluminum filter foil of $200 \mu \mathrm{m}$ thickness attenuated the high betatron flux of up to 20000 photons/eV at the peak with critical energies from 5 to $15 \mathrm{keV}$. The attenuation was necessary to enable single-shot reconstruction of the betatron spectrum by counting single-photon events [43-45]. Behind the electron spectrometer, a scintillator screen was located, oriented at $45^{\circ}$ with respect to the beam axis, to record the angularly resolved betatron profile. A 5-mm aperture in this screen allowed the transmission of the on axis betatron radiation toward the $\mathrm{x}$-ray CCD camera.

\section{EXPERIMENTAL RESULTS}

To access the beam loading regime in a controlled manner, the amount of injected charge was tuned by varying the nitrogen concentration, while keeping laser parameters and plasma density constant [9]. Applying our approach, the location, volume, and duration of the injection do not alter significantly between various dopings. Thus the electron dynamics during injection, acceleration, and extraction are predominantly influenced by the beam charge and the consequent local modification of the accelerating field, as also supported by simulations. Alternative approaches to acceleration studies with variable plasma length gas cells [46] are more challenging, because residual laser light typically damages the cell's aperture, affecting the plasma density and profile. In the data analysis for electron and betatron spectra, only shots were evaluated that showed a narrow band energy feature and were oriented on axis. Hence, we excluded spectrally modulated electron beams [47] and off-center shots that may originate from asymmetric plasma waves $[48,49]$ induced by higherorder transverse fluctuations of the laser beam or shot-toshot pointing jitter. Electron beam parameters such as maximum energy, bandwidth, divergence, and beam size at the accelerator exit are studied as a function of injected charges for a set of three plasma densities.

The effect of beam loading on the suppression of the accelerating field [37] is evident in energy $E$ and energy bandwidth $\Delta E / E$ of the accelerated electron bunches. While $E$ decreases from 400 to $300 \mathrm{MeV}$ with increasing charge [50], $\Delta E / E$ reduces reaching a minimum at $300 \pm 50 \mathrm{pC}$, as presented in Fig. 3(a). This charge value

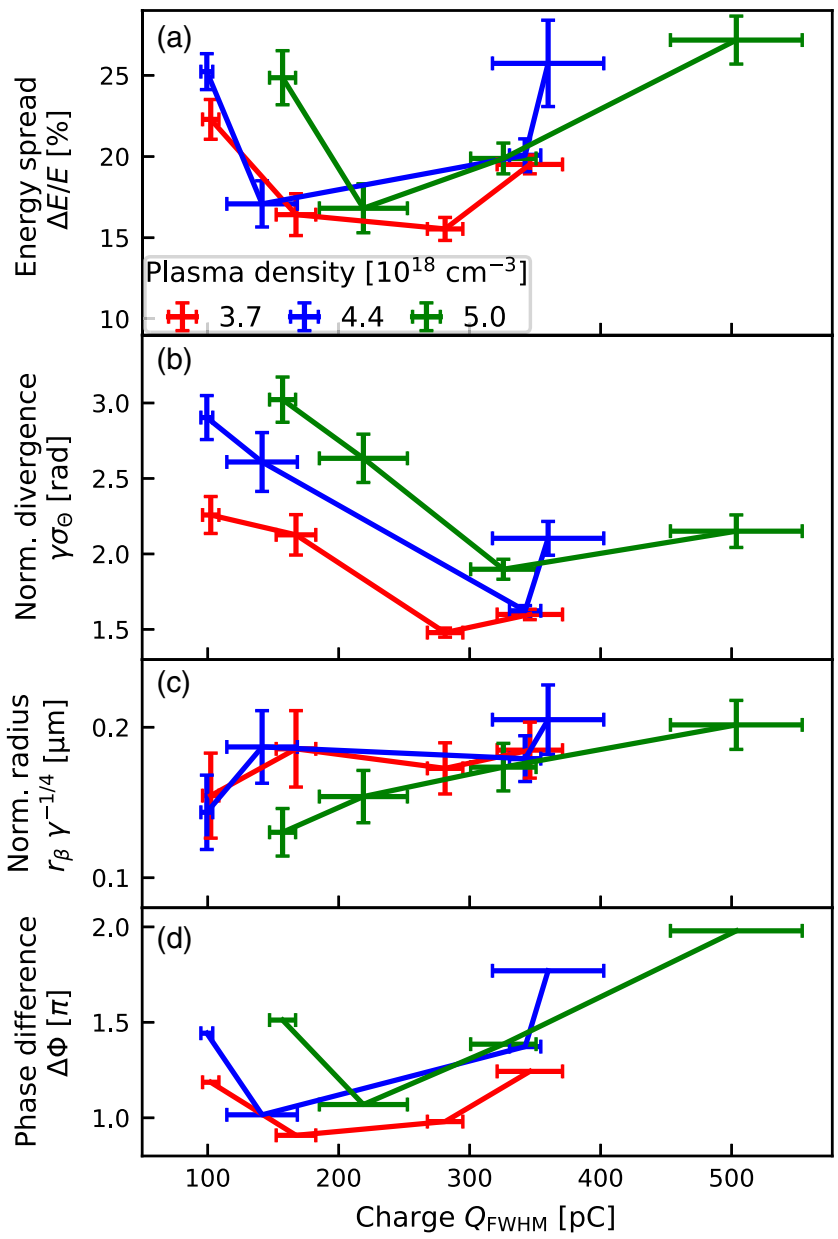

FIG. 3. Measured relative energy spread $\Delta E / E$ (a), normalized divergence $\gamma \sigma_{\Theta}$ (b), betatron radius $r_{\beta} \gamma^{-1 / 4}$, (c) and calculated betatron phase difference $\Delta \phi$ according to Eq. (2) (d) obtained from electron bunches with different charges and at three different plasma densities, $3.7 \times 10^{18} \mathrm{~cm}^{-3}$ (red line), $4.4 \times$ $10^{18} \mathrm{~cm}^{-3}$ (blue line), and $5.0 \times 10^{18} \mathrm{~cm}^{-3}$ (green line). Every data point represents the average of up to 15 shots with constant experimental parameters and the error bars denote the standard error of the mean. The error bars in (c) include the systematic error obtained from sensitivity analysis [14]. The maximum beam energy ranges from $300 \mathrm{MeV}$ to $450 \mathrm{MeV}$. 
is referred to as matched beam loading [10] for our experimental parameters. Injection of less or more charges from this optimum results in field gradients that can lead to a broadening of the bunch energy bandwidth.

In order to investigate the transverse electron dynamics, the geometric divergence of the beam $\sigma_{\Theta}$ is extracted from the nondispersive plane of the electron spectrometer after having left the plasma accelerator. Defocusing effects of the magnetic fields are corrected during data analysis. Figure 3(b) shows the normalized beam divergence $\gamma \sigma_{\Theta}$ for various plasma densities where $\gamma \approx E /\left(m_{e} c^{2}\right)$ is the relativistic Lorentz factor, $m_{e}$ and $c$ are the electron mass and the speed of light, respectively. In particular, a similar charge-dependent trend as in $\Delta E / E$ is clearly seen. At matched beam loading, a drop of up to $20 \%$ of the normalized beam divergence is measured alongside the minimal energy bandwidth. This consistently occurs for all plasma densities, which suggest that the accelerator geometry, i.e., cavity size, plasma wavelength, and the plasma down-ramp length, does not play a dominant role in the measured divergence reduction. In addition, deduced from the measured betatron spectrum $[32,44]$, the betatron source size and thus the electron beam radius is compared for the measured charges at the end of the accelerator. Figure 3(c) presents the reconstructed betatron source radii $r_{\beta} \gamma^{-1 / 4}$, where the $\gamma^{-1 / 4}$ factor normalizes differences due to the relativistic acceleration [2]. Note that the betatron radius exhibits no extremum at $300 \mathrm{pC}$ but maintains a micrometer-small source size. For this spatially compact bunch, the normalized divergence reduces at the matched beam loading charge. This indicates that space charge does not dominate the beam divergence during acceleration in the plasma as well as during propagation in the drift space, as confirmed below by simulations. Otherwise, a monotonic increase of the beam divergence with charge should be expected.

In order to explain the reduced normalized divergence observed at matched beam loading, we investigate the beam decoherence. The degree of decoherence is defined by the maximum difference of the betatron phase $\Delta \phi=\phi_{\max }-$ $\phi_{\min }$. As shown in Fig. 1, an electron in a slice $i$ with energy $\gamma_{i}$ rotates in the transverse phase space $\left(x, p_{x}\right)$ with the energydependent betatron frequency $\omega_{\beta, i}=\omega_{p} / \sqrt{2 \gamma_{i}}$, where $\omega_{p}$ is the plasma frequency. It thereby gains the phase $\phi_{i}=$ $\int_{0}^{l_{\text {acc }}} d t \omega_{\beta, i}$ over the acceleration length $l_{\mathrm{acc}, i}$. Assuming this electron experiences a constant accelerating field $E_{z, i}$, it gains the energy $\gamma_{i}=\gamma_{0}+\gamma_{i}^{\prime} l_{\mathrm{acc}, i}$ with $\gamma_{i}^{\prime}=e E_{z, i} / m_{e} c^{2}$ denoting the Lorentz factor gain per distance. The integration of $\phi_{i}$ then yields

$$
\phi_{i}=\frac{\sqrt{2} k_{p}}{\gamma_{i}^{\prime}} \sqrt{\gamma_{0}+\gamma_{i}^{\prime} l_{\mathrm{acc}, i}}+\phi_{0}
$$

where $k_{p}=\omega_{p} / c, \gamma_{0}$, and $\phi_{0}$ are the plasma wave number, initial electron energy, and initial phase, respectively. For full decoherence $(\Delta \phi \geq \pi)$, the injected electrons fill the entire phase space available during betatron oscillation and temporal minima in divergence cannot be reached anymore. Assuming an injection duration much smaller than the inverse betatron frequency, reaching full decoherence requires an acceleration length of $l_{\mathrm{dc}}=2.35 \lambda_{\beta}\langle E\rangle / \Delta E$ [34,51], with $\lambda_{\beta}=\sqrt{2 \gamma} \lambda_{p}$ being the betatron wavelength, $\lambda_{p}=2 \pi c / \omega_{p}$ is the plasma wavelength, and $\langle E\rangle$ is the average energy of the peaked spectrum at the end of acceleration. For the electrons observed in experiment, $l_{\mathrm{dc}}$ is longer than two millimeters and thus longer than the acceleration length $l_{\text {acc }}=1.6 \mathrm{~mm}$. Therefore, the accelerated electron bunches will not fully experience beam decoherence assuming an instantaneous injection. When further assuming that electrons are injected at rest $\left(\gamma_{0}=1\right.$, $\left.\Delta \phi_{0}=0\right)$ and that the energy spread is small $(\Delta E \ll\langle E\rangle)$, an estimation of the phase difference $\Delta \phi$ can be determined solely on values measured in the experiment [35]:

$$
\Delta \phi \approx \frac{\sqrt{2} k_{p}}{\gamma_{\max }^{\prime}} \sqrt{1+\gamma_{\max }^{\prime} l_{\mathrm{acc}}}-\frac{\sqrt{2} k_{p}}{\gamma_{\min }^{\prime}} \sqrt{1+\gamma_{\min }^{\prime} l_{\mathrm{acc}}},
$$

where $\gamma_{\min / \max } \propto E_{z, \min / \max }$ are deduced from the measured energy gain and spread. Representing Eq. (2), Fig. 3(d) presents the phase difference $\Delta \phi$ as a function of charge, exhibiting a minimum in the vicinity of the matched beam loading condition as described by the charge corresponding to minimal energy spread. This similarity in the behavior of $\Delta \phi$ and beam divergence $\gamma \sigma_{\Theta}$ suggests that a reduction of the phase difference below the decoherence threshold $(\Delta \phi=\pi)$ under optimal beam loading conditions could be responsible for the observed divergence minimum. For a phase advance $\Delta \phi_{0} \neq 0$ accumulated during injection, highly relativistic energies $\left(\gamma^{\prime} l_{\text {acc }} \gg 1\right)$ and small relative energy spreads $\left(\Delta \gamma^{\prime} \ll \bar{\gamma}^{\prime}\right)$, Eq. (2) can be simplified to

$$
\Delta \phi \approx \Delta \phi_{0}-\Delta \phi_{\text {beam }}=\Delta \phi_{0}-\frac{k_{p} \Delta \gamma^{\prime}}{\sqrt{2} \bar{\gamma}^{\prime 2}} \sqrt{\bar{\gamma}^{\prime} l_{\text {acc }}}
$$

with $\quad \Delta \gamma^{\prime}=\gamma_{\max }^{\prime}-\gamma_{\min }^{\prime} \quad$ and $\quad \bar{\gamma}^{\prime}=\left(\gamma_{\max }^{\prime}+\gamma_{\min }^{\prime}\right) / 2$. Equation (3) indicates that $\Delta \phi_{0}$ can be compensated by careful tuning of energy and energy spread. It directly expresses the scaling of two main quantities of beam loading: the $\bar{\gamma}^{\prime}$ is proportional to the mean accelerating field and $\Delta \gamma^{\prime}$ is proportional to the accelerating field gradient, which leads to energy spread during acceleration. The scaling of Eq. (3) shows two trends. First, at injected charges far below beam loading, the mean accelerating field is at maximum. When increasing the injected charge toward beam loading, $\Delta \gamma^{\prime}$ and $\bar{\gamma}^{\prime}$ decrease. Second, while $\phi_{\text {beam }}$ vanishes for ideal beam loading $\left(\Delta \gamma^{\prime}=0\right)$, the counteracting scalings of $\bar{\gamma}^{\prime}$ and $\Delta \gamma^{\prime}$ indicate the existence of a maximum in $\Delta \phi_{\text {beam }}$ before optimal beam loading is reached. The detailed phase progression with injected charge depends on the beam loading dynamics and can be evaluated from experimental data on the 
final electron energy and energy spread varying with injected charge.

In Sec. IV, the hypothesis of minimized phase spread will be further investigated. In particular, the previous model assumptions will be expanded by dedicated simulations focusing on the injection dynamics and subsequent phase advance during acceleration.

\section{SIMULATIONS}

For testing the decoherence hypothesis, we performed rigorous three-dimensional PIC simulations with the PIConGPU code [52,53] modeling realistic experimental parameters, as explained in Ref. [50]. This setup can be found under Ref. [54]. As in the experiment, to inject more charge into the wakefield, the nitrogen doping concentration was increased for fixed laser-plasma parameters. Moreover, a particle identification scheme implemented in the code allows for tracking the nitrogen $K$-shell electrons, source of the self-truncated ionization injection injected bunch, through the simulation. For beam propagation in vacuum toward a virtual $2 \mathrm{D}$ spectrometer, the general particle tracer (GPT) code [55] was deployed which is fed by the PIC particle data at the accelerator exit using an openPMD-based particle reduction [56,57]. This start-toend simulation enables us to investigate the complete sixdimensional phase space dynamics in detail, starting from electron injection and trapping, acceleration, until extraction and vacuum propagation.

\section{A. Injection}

Figure 4(a) presents the injection rate, i.e., number of electrons trapped from the nitrogen $K$-shell as the laser propagates along the $z$ axis, for nitrogen doping concentrations of $0.25 \%$ (blue line), $0.5 \%$ (orange line), $1.0 \%$ (green line), and $3.0 \%$ (red line). Once trapped in the wakefield, these electrons are accelerated to form a high energy and small bandwidth bunch at the accelerator exit, where the total accumulated charges of 30,50, 100, and $150 \mathrm{pC}$ within FWHM energy bandwidth are generated from the lowest to the highest doping, respectively. The comparatively lower charges obtained here than in the experiment can be attributed to idealized laser pulse modeling. The resulted higher electron energy in simulations imply that electrons are injected and accelerated at higher fields. For a given plasma density, the optimal loading charge can strongly depend on the accelerating field and plasma cavity [37]. Idealized, spatially limited laser pulses can cause stronger acceleration and a smaller cavity, thus reducing the beam loading charge in simulation. Also, note that the longitudinal electron beam profiles in the experiment do not necessarily follow the ideal trapezoidal charge distribution [37] and, similarly, the measurements can deviate from the simulation results which assume more ideal laser pulses. Thus, the total
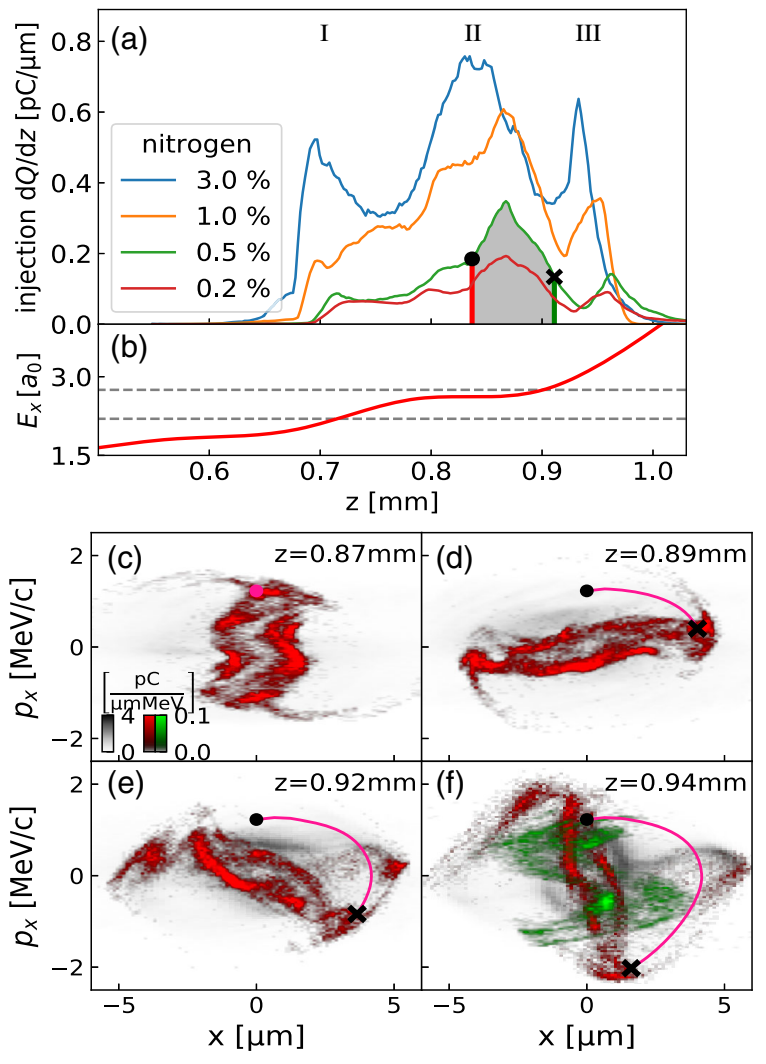

FIG. 4. $K$-shell ionization and electron injection: (a) shows the injection rate $d Q / d z$ of all trapped electrons and three distinct injections (I, II, and III). (b) indicates the evolution of the laser peak intensity $a_{0}$. The gray dashed lines indicate the ionization threshold for the first and second nitrogen $K$-shell electrons. (c)-(f) presents the electron transverse phase space distribution $\left(x, p_{x}\right)$ in the laser polarization plane for the $0.5 \%$ nitrogen doping at $z=0.87,0.89,0.92$, and $0.94 \mathrm{~mm}$. The gray scale represents macroparticles from region II marked in (a). The red and green scale represent slices of earliest and latest injected electrons as indicated in (a), respectively. Extracted from the simulation, the red line represents the path of sample macroparticles that are injected (dot) in the earliest slice at (c) and rotation until the actual time (cross) shown in (d), (e), and (f). The simulations were performed for a plasma density of $4.4 \times 10^{18} \mathrm{~cm}^{-3}$.

beam charge can vary despite achieving similar minimal electron beam energy spreads at optimal beam loading. Hence in our experiment-to-simulation comparisons, we show how the observables scale relative to a working point at optimal beam loading rather than the same absolute beam charges.

At the end of the plasma channel, the macroparticles in the FWHM of the energy are selected and traced back to the injection to deduce $d Q / d z$. While the total injection length is about $300 \mathrm{~mm}$ for all dopings, three distinct regions of high injection rate $d Q / d z$ with an injection length of less than $100 \mathrm{~mm}$ can be identified. Particularly, the majority of electrons is injected within the second region peaked at 
$z \approx 0.84 \mathrm{~mm}$ and dominates the transverse beam dynamics. The analysis of the betatron phase will focus on this group of electrons.

The $d Q / d z$ peaks are closely linked to the evolution of the laser peak intensity, as presented in Fig. 4(b). During propagation inside the plasma, the peak intensity of the laser pulse increases due to self-focusing which consequently impacts the shape of the wakefield. When the intensity exceeds the ionization threshold of the first nitrogen $K$-shell and the trapping condition is satisfied [58-60], electrons from this shell are injected with the rate presented by the regions I and II. While the laser pulse further self-focuses, the peak intensity increases and this eventually leads to the injection of the second $K$-shell electrons in the region III. Injection self-terminates once the trapping condition is lost due to the wakefield evolution.

Concentrating on region II, Figs. 4(c)-4(f) show the transverse phase space $\left(x, p_{x}\right)$ on the laser polarization plane for the $0.5 \%$ doping during injection, i.e., at $z \approx 0.87-0.94 \mathrm{~mm}$. Comparing the phase space of earliest injected electrons (red) to the last (green), the red slice has rotated by almost $\pi$ before the green slice was injected at the end of region II. The injection duration leads to an initial phase advance of $\Delta \phi \approx \pi$ and thus acceleration starts with a fully decoherent bunch. As an example, the magenta line indicates the path of a small sample of macroparticles traced by the simulation. The same injection evolution also applies for other doping concentrations.

\section{B. Acceleration}

During acceleration, the focusing forces of the wakefield ensure that the beam stays close to the axis. Figure 5(a) illustrates the focusing field map of the wakefield at $z=$ $1.7 \mathrm{~mm}$ which is loaded with a relatively high charge of $100 \mathrm{pC}$, generated by the $1 \%$ nitrogen doping. The blue and orange line outs in Fig. 5(b) are sampling the focusing force $F$ at different longitudinal positions $\zeta=z-c t$, i.e., in the front of the bunch and at the bunch center, respectively,
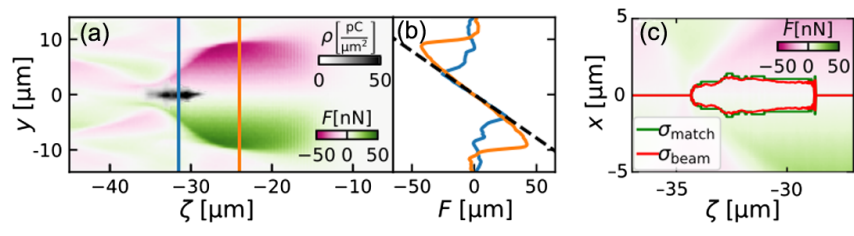

FIG. 5. Beam loading in PIC simulation: (a) illustrates the focusing field map $F=e(E-c B)$ for an injected charge of 100 $\mathrm{p} \mathrm{C}$ at $z=1.7 \mathrm{~mm}$. The density of injected electrons and the focusing force are indicated by the gray and color scale at the bottom right, respectively, (b) presents two line outs of the focusing force in front of the bunch (orange) and at the bunch (blue) emphasizing its linearity. The black dashed line in (b) indicates the theoretically predicted value $-E_{0} / 2 k_{p} r$ [61], and (c) shows the beam size $\sigma_{\text {beam }}(\zeta)$ (red line) and the matched beam size $\sigma_{\text {match }}(\zeta)$ (green line) after injection $(z=1.1 \mathrm{~mm})$. emphasizing the linearity and independence of $F$ on space charge. Despite that the accelerating field being locally modified due to the beam loading, the focusing remains radially linear and longitudinally constant. This important feature can preserve the transverse slice emittance for the acceleration of high-quality beams and thereby allows to study the beam evolution, i.e., beam matching and decoherence. In the case of beam matching, $F$ balances beam defocusing driven by finite emittance and the beam size remains constant along the acceleration distance [30]. An unmatched beam oscillates in beam size similar to a not fully decoherent bunch. Figure 5(c) shows the rms beam envelope of the $100 \mathrm{pC}$ charge case confined within the wakefield focusing field $F$ at $\mathrm{z}=1.0 \mathrm{~mm}$. The red line represents the beam size $\sigma_{\text {beam }}(\zeta)$ and the green line shows the matched beam size $\sigma_{\text {match }}(\zeta)$ given by [30]

$$
\sigma_{\text {match }}(\zeta)=\gamma\left(\frac{m_{e} c^{2}}{\epsilon_{n}(\zeta) F(\zeta)}\right)^{1 / 3},
$$

where $\epsilon_{n}(\zeta)$ is the rms normalized transverse emittance of the particle distribution at $\zeta$. Even for large dopings as shown for the $100 \mathrm{pC}$ case in Fig. 5(c), the beam matching condition is already satisfied at the beginning of the acceleration and maintained during the adiabatic acceleration $\left(\sigma_{\text {beam }} \propto \gamma^{-1 / 4}\right)$. Together, the initially matched beam and the decoherent phase space distribution immediately after injection allow us to study the beam decoherence during the subsequent acceleration process since the reversal of beam decoherence can be attributed solely to the acceleration gradient set by the beam loading.

Figures 6(a) and 6(b) exemplify the evolution of the transverse momentum and beam envelope for the bunch charge of $50 \mathrm{pC}$ from injection until extraction to vacuum. Periodic modulations in the electron beam size are visible, indicating that the bunch is partially coherent during acceleration [35] consistent with the estimation of the decoherence length $l_{\mathrm{dc}}$. Furthermore, in Fig. 6(c), the red and green lines represent the orbits of selected particles from the first and last slice of the region II in Fig. 4(a), respectively. As shown in Fig. 6(d), the betatron phase $\phi$ and difference $\Delta \phi$ are calculated from $\left(x, p_{x}\right)$. After injection, the difference in phase rapidly reduces within $500 \mathrm{~mm}$ from an almost fully decoherent bunch $(\Delta \phi \approx \pi)$ to a partially coherent bunch $(\Delta \phi \approx \pi / 4)$. This is a result of the highly relativistic energy during acceleration which reduces $\omega_{\beta}$, the rate of increase $\dot{\phi}$, and thus the relative rotation speed of the slices in $\left(x, p_{x}\right)$ with respect to each other. Partial coherence $(\Delta \phi<\pi)$ typically decreases the divergence in the case of a phase space ellipse aligned along the spatial coordinate. Therefore, the bunch partly restores coherence during acceleration while starting fully decoherent at injection. Summarizing the simulation results at the accelerator exit, Fig. 6(e) plots the energy bandwidth where the simulated matched beam loading is obtained at 

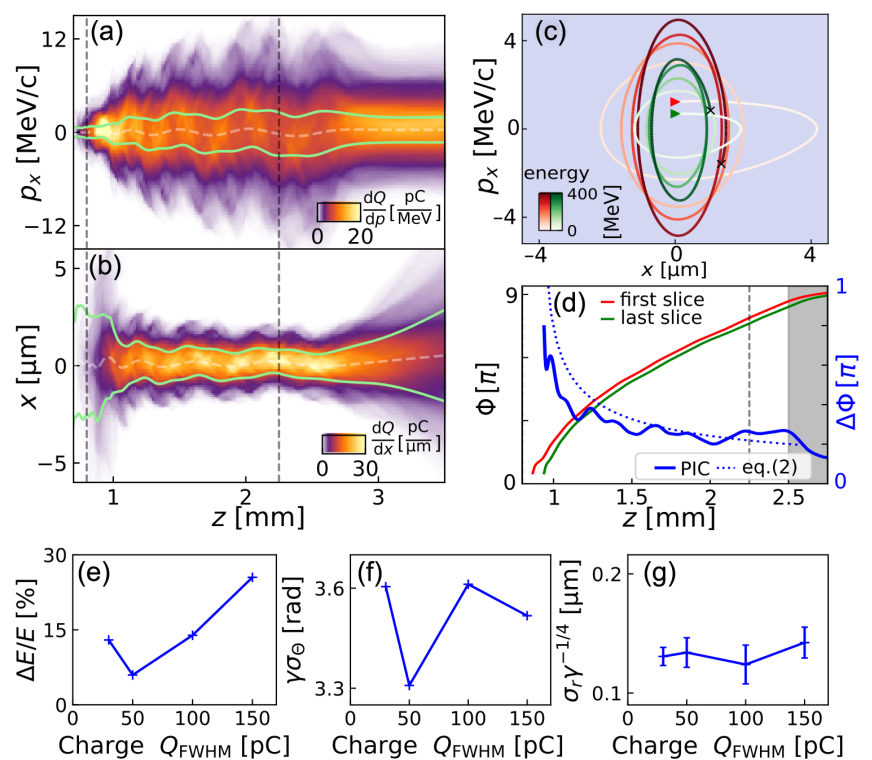

FIG. 6. PIC particle tracking for $50 \mathrm{pC}$ charge, (a) and (b) showcase the evolution of transverse momentum $p_{x}$ and coordinate $x$, respectively. The green line indicates one standard deviation $\sigma_{p}(z)$ and $\sigma_{x}(z)$. The vertical gray dashed lines indicate the plasma density up- and down-ramp. In (c), the red and green line represent particle orbits in $\left(x, p_{x}\right)$ from the earlier and later injected slice in Fig. 4(c). (d) shows the phase $\phi=$ $\arctan p_{x} / x \gamma^{1 / 2}$ of the two orbits from (c). The gray shaded area $z>2.5 \mathrm{~mm}$ indicates the field-free space outside the plasma accelerator. The thick blue line shows the phase difference $\Delta \phi$, which indicates a decreasing decoherence during acceleration. The dashed blue line is calculated with Eq. (2) using an averaged $\gamma^{\prime}$. The vertical gray dashed line indicates the beginning of the plasma density down-ramp. Close to the end of acceleration $(z=2.0 \mathrm{~mm})$, (e), (f), and $(\mathrm{g})$ present the beam energy bandwidth $\Delta E / E$, the normalized divergence $\gamma \sigma_{\Theta}$, and the normalized beam radius $\sigma_{r} \gamma^{-1 / 4}$, respectively. Error bars indicate one standard deviation in $(\mathrm{g})$.

$50 \mathrm{pC}$ charge. Defined by the transverse momentum spread $\sigma_{p}^{f}$ at the plasma exit, the normalized divergence $\gamma \sigma_{\Theta}=$ $\sigma_{p}^{f} /\left(m_{e} c\right)$ reaches a minimum at this matched beam loading charge, shown in Fig. 6(f), consistent with the experiment. In Fig. 6(g), it can be seen that the normalized beam size $\sigma_{r} \gamma^{-1 / 4}$ does not increase significantly with charge and is about $0.15 \mathrm{~mm}$ which is in agreement with the experimental measurements.

\section{Extraction and vacuum propagation}

In our experiment, accelerated electrons are detected after propagation in a vacuum much longer than the plasma channel and space charge effects could affect the measured beam parameters. For an efficient simulation of the beam dynamics in such a drift space, particle tracing simulations were performed with the GPT code [55], taking the space charge effect via the space charge three-dimensional model into account. From the previously performed PIC
TABLE I. Space charge effects in the drift space: beam parameters after $50 \mathrm{~cm}$ of free propagation simulated using GPT.

\begin{tabular}{lccccc}
\hline \hline $\begin{array}{l}\text { Charge } \\
{[\mathrm{pC}]}\end{array}$ & $\begin{array}{c}\text { Normalized } \\
\text { divergence } \\
{[\mathrm{rad}]}\end{array}$ & $\begin{array}{c}\text { Geometrical } \\
\text { divergence } \\
{[\mathrm{mrad}]}\end{array}$ & $\begin{array}{c}\text { Beam } \\
\text { size } \\
{[\mathrm{mm}]}\end{array}$ & $\begin{array}{c}\text { Average } \\
\text { energy } \\
{[\mathrm{MeV}]}\end{array}$ & $\begin{array}{c}\text { Energy } \\
\text { spread } \\
{[\mathrm{MeV}]}\end{array}$ \\
\hline 100 & 3.29 & 4.4 & 2.2 & 380 & 20 \\
300 & 3.34 & 4.4 & 2.2 & 389 & 22 \\
500 & 3.49 & 4.4 & 2.2 & 397 & 24.5 \\
\hline \hline
\end{tabular}

simulations as shown in Fig. 6, the macroparticles at the plasma exit were extracted, reduced to a set of 5000 and fed into GPT [57]. The charge of this particle distribution was upscaled to 100,300 , and $500 \mathrm{pC}$ in order to mimic experimentally observed maximal charges and to accurately study the space charge effect. Reflecting experimental conditions, these macroparticles were tracked from the plasma exit throughout a $50 \mathrm{~cm}$ long drift space. Table I lists the transverse beam parameters of a typical electron bunch from these simulations. Energy and energy spread increase by 5\%-20\% for higher bunch charges, respectively. The beam size and geometrical divergence after propagation seem to be charge independent and the normalized divergence monotonically increases by about $10 \%$ when the charge increases by a factor of five. For a high-bunch charge, electrons are accelerated by the Coulomb repulsion which causes electrons to gain transverse and longitudinal momentum [62]. The monotonic increase is in contrast to the nonlinear charge-dependent betatron decoherence described before. Thus, space charge effects during propagation in the drift space cannot explain the minima in the normalized divergence.

\section{SUMMARY}

In summary, we experimentally demonstrated that matched beam loading in a laser-wakefield accelerator, identified via its characteristic charge-dependent minimum in beam energy spread, additionally yields a minimum in normalized beam divergence. The experiment relied on control over the injected charge in the self-truncated ionization injection regime and on monitoring the beam diameter inside the plasma via betatron $\mathrm{x}$-ray spectroscopy. Combined with simplified analytical modeling of the betatron phase advance per given acceleration length, the experiment suggests that the observation of reduced divergence is a result of the suppression of betatron phase decoherence. Detailed studies of the complete six-dimensional phase space dynamics of injected electrons using high-resolution PIC simulations revealed that despite the injection extending over the full initial betatron period, the decoherent bunch is partially restoring its betatron coherence during acceleration in the beam-loaded field.

This finding substantiates the importance of operating plasma accelerators in the matched beam loading regime 
for the simultaneous optimization of beam charge and transverse emittance. High-beam quality and in particular low divergence is essential for the realization of the next generation of high-brightness light sources and facilitates beam transport to subsequent stages or insertion devices like undulators. In combination with the high-peak currents of the beam-loaded regime, this demonstration of optimized electron beam quality opens a pathway to the realization of high-brightness radiation sources.

\section{ACKNOWLEDGMENTS}

The authors gratefully acknowledge the team from DRACO as well as T. Cowan, M. Downer, J. Grenzer, A. Laso García, K. Steiniger, A. Wagner, and R. Zgadzaj for their support. This work was partly supported by EuCARD2, funding under Grant Agreement No. 312453, by HZDR under program Matter and Technology, and by the Center of Advanced Systems Understanding (CASUS) which is financed by Germany's Federal Ministry of Education and Research (BMBF) and by the Saxon Ministry for Science, Culture and Tourism (SMWK) with tax funds on the basis of the budget approved by the Saxon State Parliament. The authors gratefully acknowledge the GWK support for funding this project by providing computing time through the Center for Information Services and HPC (ZIH) at TU Dresden on the HRSK-II.

[1] E. Esarey, C. B. Schroeder, and W. P. Leemans, Physics of laser-driven plasma-based electron accelerators, Rev. Mod. Phys. 81, 1229 (2009).

[2] M. C. Downer, R. Zgadzaj, A. Debus, U. Schramm, and M. C. Kaluza, Diagnostics for plasma-based electron accelerators, Rev. Mod. Phys. 90, 035002 (2018).

[3] A. J. Gonsalves et al., Petawatt Laser Guiding and Electron Beam Acceleration to $8 \mathrm{GeV}$ in a Laser-Heated Capillary Discharge Waveguide, Phys. Rev. Lett. 122, 084801 (2019).

[4] C. G. R. Geddes, K. Nakamura, G. R. Plateau, C. Toth, E. Cormier-Michel, E. Esarey, C. B. Schroeder, J. R. Cary, and W. P. Leemans, Plasma-Density-Gradient Injection of Low Absolute-Momentum-Spread Electron Bunches, Phys. Rev. Lett. 100, 215004 (2008).

[5] J. Osterhoff, A. Popp, Z. Major, B. Marx, T. P. RowlandsRees, M. Fuchs, M. Geissler, R. Hörlein, B. Hidding, S. Becker, E. A. Peralta, U. Schramm, F. Grüner, D. Habs, F. Krausz, S. M. Hooker, and S. Karsch, Generation of Stable, Low-Divergence Electron Beams by Laser-Wakefield Acceleration in a Steady-State-Flow Gas Cell, Phys. Rev. Lett. 101, 085002 (2008).

[6] C. Rechatin, J. Faure, A. Ben-Ismail, J. Lim, R. Fitour, A. Specka, H. Videau, A. Tafzi, F. Burgy, and V. Malka, Controlling the Phase-Space Volume of Injected Electrons in a Laser-Plasma Accelerator, Phys. Rev. Lett. 102, 164801 (2009).
[7] A. J. Gonsalves, K. Nakamura, C. Lin, D. Panasenko, S. Shiraishi, T. Sokollik, C. Benedetti, C. B. Schroeder, C. G. R. Geddes, J. van Tilborg, J. Osterhoff, E. Esarey, C. Toth, and W. P. Leemans, Tunable laser plasma accelerator based on longitudinal density tailoring, Nat. Phys. 7, 862 (2011).

[8] A. Buck, J. Wenz, J. Xu, K. Khrennikov, K. Schmid, M. Heigoldt, J. M. Mikhailova, M. Geissler, B. Shen, F. Krausz, S. Karsch, and L. Veisz, Shock-Front Injector for High-Quality Laser-Plasma Acceleration, Phys. Rev. Lett. 110, 185006 (2013).

[9] A. Irman, J. P. Couperus, A. Debus, A. Köhler, J. M. Krämer, R. Pausch, O. Zarini, and U. Schramm, Improved performance of laser wakefield acceleration by tailored self-truncated ionization injection, Plasma Phys. Controlled Fusion 60, 044015 (2018).

[10] J. P. Couperus, R. Pausch, A. Köhler, O. Zarini, J. M. Krämer, M. Garten, A. Huebl, R. Gebhardt, U. Helbig, S. Bock, K. Zeil, A. Debus, M. Bussmann, U. Schramm, and A. Irman, Demonstration of a beam loaded nanocoulomb-class laser wakefield accelerator, Nat. Commun. 8, 487 (2017).

[11] J. Götzfried, A. Döpp, M. F. Gilljohann, F. M. Foerster, H. Ding, S. Schindler, G. Schilling, A. Buck, L. Veisz, and S. Karsch, Physics of High-Charge Electron Beams in LaserPlasma Wakefields, Phys. Rev. X 10, 041015 (2020).

[12] M. Kirchen, S. Jalas, P. Messner, P Winkler, T. Eichner, L. Hübner, T. Hülsenbusch, L. Jeppe, T. Parikh, M. Schnepp, and A. Maier, Optimal Beam Loading in a Laser-Plasma Accelerator, Phys. Rev. Lett. 126, 174801 (2021).

[13] Y. Glinec, J. Faure, A. Norlin, A. Pukhov, and V. Malka, Observation of Fine Structures in Laser-Driven Electron Beams Using Coherent Transition Radiation, Phys. Rev. Lett. 98, 194801 (2007).

[14] A. D. Debus et al., Electron Bunch Length Measurements from Laser-Accelerated Electrons Using Single-Shot THz Time-Domain Interferometry, Phys. Rev. Lett. 104, 084802 (2010).

[15] O. Lundh, J. Lim, C. Rechatin, L. Ammoura, A. Ben-Ismail, X. Davoine, G. Gallot, J.-P. Goddet, E. Lefebvre, V. Malka, and J. Faure, Few femtosecond, few kiloampere electron bunch produced by a laser-plasma accelerator, Nat. Phys. 7, 219 (2011).

[16] O. Zarini, A. Köhler, J. Couperus, R. Pausch, T. Kurz, S. Schöbel, H. Meißner, M. Bussmann, U. Schramm, A. Irman, and A. Debus, Advanced methods for temporal reconstruction of modulated electron bunches, in Proceedings of the IEEE Advanced Accelerator Concepts Workshop (AAC) (2018), pp. 1-5, Technical Report No. 978-1-5386-7721-6.

[17] O. Zarini, J. C. Cabadağ et al., Multioctave high-dynamic range optical spectrometer for single-pulse diagnostic applications, [Phys. Rev. Accel. Beams (to be published)], 2021.

[18] B. Green et al., High-field high-repetition-rate sources for the coherent $\mathrm{THz}$ control of matter, Sci. Rep. 6, 22256 (2016).

[19] B. Hidding, T. Königstein, J. Osterholz, S. Karsch, O. Willi, and G. Pretzler, Monoenergetic Energy Doubling in a Hybrid Laser-Plasma Wakefield Accelerator, Phys. Rev. Lett. 104, 195002 (2010). 
[20] A. Martinez de la Ossa, J. Grebenyuk, T. Mehrling, L. Schaper, and J. Osterhof, High-Quality Electron Beams from Beam-Driven Plasma Accelerators by WakefieldInduced Ionization Injection, Phys. Rev. Lett. 111, 245003 (2013).

[21] T. Kurz et al., Demonstration of a compact plasma accelerator powered by laser-accelerated electron beams, Nat. Commun. 12, 2895 (2021).

[22] H.-P. Schlenvoigt, K. Haupt, A. Debus, F. Budde, O. Jäckel, S. Pfotenhauer, H. Schwoerer, E. Rohwer, J. G. Gallacher, E. Brunetti, R. P. Shanks, S. M. Wiggins, and D. A. Jaroszynski, A compact synchrotron radiation source driven by a laser-plasma wakefield accelerator, Nat. Phys. 4, 130 (2008).

[23] M. Fuchs, R. Weingartner, A. Popp, Z. Major, S. Becker, J. Osterhoff, I. Cortrie, B. Zeitler, R. Hörlein, G. D. Tsakiris, U. Schramm, T. P. Rowlands-Rees, S. M. Hooker, D. Habs, F. Krausz, S. Karsch, and F. Grüner, Laser-driven softX-ray undulator source, Nat. Phys. 5, 826 (2009).

[24] A. R. Maier, A. Meseck, S. Reiche, C. B. Schroeder, T. Seggebrock, and F. Grüner, Demonstration Scheme for a Laser-Plasma-Driven Free-Electron Laser, Phys. Rev. X 2 , 031019 (2012).

[25] Z. Huang, Y. Ding, and C. B. Schroeder, Compact X-Ray Free-Electron Laser from a Laser-Plasma Accelerator Using a Transverse-Gradient Undulator, Phys. Rev. Lett. 109, 204801 (2012).

[26] K. Steiniger, M. Bussmann, R. Pausch, T. Cowan, A. Irman, A. Jochmann, R. Sauerbrey, U. Schramm, and A. Debus, Optical free-electron lasers with traveling-wave Thomson-scattering, J. Phys. B 47, 234011 (2014).

[27] T. Eichner, F. Grüner, S. Becker, M. Fuchs, D. Habs, R. Weingartner, U. Schramm, H. Backe, P. Kunz, and W. Lauth, Miniature magnetic devices for laser-based, tabletop free-electron lasers, Phys. Rev. ST Accel. Beams 10, 082401 (2007).

[28] T. André et al., Control of laser plasma accelerated electrons for light sources, Nat. Commun. 9, 1334 (2018).

[29] A. G. Khachatryan, A. Irman, F. A. Van Goor, and K. J. Boller, Femtosecond electron-bunch dynamics in laser wakefields and vacuum, Phys. Rev. ST Accel. Beams 10, 121301 (2007).

[30] E. Esarey, B. Shadwick, P. Catravas, and W. Leemans, Synchrotron radiation from electron beams in plasmafocusing channels, Phys. Rev. E 65, 056505 (2002).

[31] F. Albert, R. Shah, K. T. Phuoc, R. Fitour, F. Burgy, J.-P. Rousseau, A. Tafzi, D. Douillet, T. Lefrou, and A. Rousse, Betatron oscillations of electrons accelerated in laser wakefields characterized by spectral x-ray analysis, Phys. Rev. E 77, 056402 (2008).

[32] M. Schnell, A. Sävert, B. Landgraf, M. Reuter, M. Nicolai, O. Jäckel, C. Peth, T. Thiele, O. Jansen, A. Pukhov, O. Willi, M.C. Kaluza, and C. Spielmann, Deducing the Electron-Beam Diameter in a Laser-Plasma Accelerator Using X-Ray Betatron Radiation, Phys. Rev. Lett. 108, 075001 (2012).

[33] S. Corde, K. T. Phuoc, G. Lambert, R. Fitour, V. Malka, A. Rousse, A. Beck, and E. Lefebvre, Femtosecond $\mathrm{x}$ rays from laser-plasma accelerators, Rev. Mod. Phys. 85, 1 (2013).
[34] T. Mehrling, J. Grebenyuk, F. S. Tsung, K. Floettmann, and J. Osterhoff, Transverse emittance growth in staged laserwakefield acceleration, Phys. Rev. ST Accel. Beams 15, 111303 (2012).

[35] X. L. Xu, J. F. Hua, F. Li, C. J. Zhang, L. X. Yan, Y. C. Du, W. H. Huang, H. B. Chen, C. X. Tang, W. Lu, P. Yu, W. An, C. Joshi, and W.B. Mori, Phase-Space Dynamics of Ionization Injection in Plasma-Based Accelerators, Phys. Rev. Lett. 112, 035003 (2014).

[36] T. Katsouleas, S. Wilks, J. M. Dawson, P. Chen, and J. J. $\mathrm{Su}$, Beam loading in plasma waves, Part. Accel. 22, 210 (1987), http://inis.iaea.org/search/search.aspx?orig_q=RN: 18090527.

[37] M. Tzoufras, W. Lu, F. S. Tsung, C. Huang, W. B. Mori, T. Katsouleas, J. Vieira, R. A. Fonseca, and L. O. Silva, Beam Loading in the Nonlinear Regime of Plasma-Based Acceleration, Phys. Rev. Lett. 101, 145002 (2008).

[38] U. Schramm et al., First results with the novel Petawatt laser acceleration facility in Dresden, J. Phys. 874, 012028 (2017).

[39] J.P. Couperus, A. Köhler, T. A. W. Wolterink, A. Jochmann, O. Zarini, H. M. J. Bastiaens, K. J. Boller, A. Irman, and U. Schramm, Tomographic characterisation of gas-jet targets for laser wakefield acceleration, Nucl. Instrum. Methods Phys. Res., Sect. A 830, 504 (2016).

[40] M. Zeng, M. Chen, Z. M. Sheng, W. B. Mori, and J. Zhang, Self-truncated ionization injection and consequent monoenergetic electron bunches in laser wakefield acceleration, Phys. Plasmas 21, 030701 (2014).

[41] M. Mirzaie, S. Li, M. Zeng, N. A. M. Hafz, M. Chen, G. Y. Li, and Q. J. Zhu, Demonstration of self-truncated ionization injection for GeV electron beams, Sci. Rep. 5, 14659 (2015).

[42] T. Kurz, J. P. Couperus, J. M. Krämer, H. Ding, S. Kuschel, A. Köhler, O. Zarini, D. Hollatz, D. Schinkel, R. D’Arcy, J.-P. Schwinkendorf, J. Osterhoff, A. Irman, U. Schramm, and S. Karsch, Calibration and cross-laboratory implementation of scintillating screens for electron bunch charge determination, Rev. Sci. Instrum. 89, 093303 (2018).

[43] A. Jochmann, A. Irman, M. Bussmann, J. P. Couperus, T. E. Cowan, A. D. Debus, M. Kuntzsch, K. W. D. Ledingham, U. Lehnert, R. Sauerbrey, H. P. Schlenvoigt, D. Seipt, T. Stöhlker, D. B. Thorn, S. Trotsenko, A. Wagner, and U. Schramm, High Resolution Energy-Angle Correlation Measurement of Hard X Rays from Laser-Thomson Backscattering, Phys. Rev. Lett. 111, 114803 (2013).

[44] A. Köhler, J. P. Couperus, O. Zarini, A. Jochmann, A. Irman, and U. Schramm, Single-shot betatron source size measurement from a laser-wakefield accelerator, Nucl. Instrum. Methods Phys. Res., Sect. A 829, 265 (2016).

[45] J. M. Krämer, A. Jochmann, M. Budde, M. Bussmann, J. P. Couperus, T. E. Cowan, A. Debus, A. Köhler, M. Kuntzsch, A. L. García, U. Lehnert, P. Michel, R. Pausch, O. Zarini, U. Schramm, and A. Irman, Making spectral shape measurements in inverse Compton scattering a tool for advanced diagnostic applications, Sci. Rep. 8, 1398 (2018).

[46] R. J. Shalloo et al., Automation and control of laser wakefield accelerators using Bayesian optimization, Nat. Commun. 11, 6355 (2020). 
[47] Y. Glinec, J. Faure, A. Lifschitz, J. M. Vieira, R A. Fonseca, L. O. Silva, and V. Malka, Direct observation of betatron oscillations in a laser-plasma electron accelerator, Europhys. Lett. 81, 64001 (2008).

[48] A. Popp, J. Vieira, J. Osterhoff, Z. Major, R. Hörlein, M. Fuchs, R. Weingartner, T. P. Rowlands-Rees, M. Marti, R. A. Fonseca, S. F. Martins, L. O. Silva, S. M. Hooker, F. Krausz, F. Grüner, and S. Karsch, All-Optical Steering of Laser-Wakefield-Accelerated Electron Beams, Phys. Rev. Lett. 105, 215001 (2010).

[49] D. E. Mittelberger, M. Thévenet, K. Nakamura, A. J. Gonsalves, C. Benedetti, J. Daniels, S. Steinke, R. Lehe, J.-L. Vay, C. B. Schroeder, E. Esarey, and W. P. Leemans, Laser and electron deflection from transverse asymmetries in laser-plasma accelerators, Phys. Rev. E 100, 063208 (2019).

[50] See Supplemental Material at http://link.aps.org/ supplemental/10.1103/PhysRevAccelBeams.24.091302 for experimental energy and details of PIConGPU simulations.

[51] P. Michel, C. B. Schroeder, B. A. Shadwick, E. Esarey, and W. P. Leemans, Radiative damping and electron beam dynamics in plasma-based accelerators, Phys. Rev. E 74, 026501 (2006).

[52] M. Bussmann, H. Burau, T. E. Cowan, A. Debus, A. Huebl, G. Juckeland, T. Kluge, W. E. Nagel, R. Pausch, F. Schmitt, U. Schramm, J. Schuchart, and R. Widera, Radiative signatures of the relativistic kelvin-helmholtz instability, in Proceedings of the International Conference on High Performance Computing, Networking, Storage and Analysis, SC '13 (New York, NY, USA, 2013).

[53] A. Huebl, R. Widera, B. Worpitz, R. Pausch, H. Burau, M. Garten, S. Starke, A. Grund, A. Debus, A. Matthes, S. Bastrakov, K. Steiniger, I. Göthel, S. Rudat, J. Kelling, and M. Bussmann, PIConGPU 0.4.1: Minor Bugs and Example Updates, 2018.
[54] R. Pausch, A. Köhler, S. Bastrakov, M. Bussmann, J. P. C. Cabadag, A. Irman, K. Steiniger, R. Widera, and A. Debus, LWFA PIConGPU setup for minimizing transverse phase space effects paper, 2021, https://doi.org/10.14278/rodare 892 .

[55] Pulsar Physics. General particle tracer, http://www.pulsar .nl/gpt.

[56] A. Huebl, R. Lehe, J.-L. Vay, D. P. Grote, I. Sbalzarini, S. Kuschel, D. Sagan, F. Pérez, F. Koller, and M. Bussmann, openPMD 1.1.0: Base paths for mesh- and particle- only files and updated attributes, 2018, https://doi.org/10.5281/ zenodo.1167843.

[57] Particle reduction code. https://github.com/Computational RadiationPhysics/particlel_reduction.

[58] A. Pak, K. A. Marsh, S. F. Martins, W. Lu, W. B. Mori, and C. Joshi, Injection and Trapping of Tunnel-Ionized Electrons into Laser-Produced Wakes, Phys. Rev. Lett. 104, 025003 (2010).

[59] C. McGuffey, A. G. R. Thomas, W. Schumaker, T. Matsuoka, V. Chvykov, F. J. Dollar, G. Kalintchenko, V. Yanovsky, A. Maksimchuk, K. Krushelnick, I. V. Glazyrin, and A. V. Karpeev, Ionization Induced Trapping in a Laser Wakefield Accelerator, Phys. Rev. Lett. 104, 025004 (2010).

[60] M. Chen, E. Esarey, C. B. Schroeder, C. G. R. Geddes, and W. P. Leemans, Theory of ionization-induced trapping in laser-plasma accelerators, Phys. Plasmas 19, 033101 (2012).

[61] W. Lu, M. Tzoufras, C. Joshi, F. S. Tsung, W. B. Mori, J. Vieira, R. A. Fonseca, and L. O. Silva, Generating multigev electron bunches using single stage laser wakefield acceleration in a 3D nonlinear regime, Phys. Rev. ST Accel. Beams 10, 061301 (2007).

[62] F. Grüner, C. B. Schroeder, A. R. Maier, S. Becker, and J. M. Mikhailova, Space-charge effects in ultrahigh current electron bunches generated by laser-plasma accelerators, Phys. Rev. ST Accel. Beams 12, 020701 (2009). 